\title{
The effect of vertex or edge deletion on the metric dimension of graphs
}

\author{
Linda Eroh, Paul Feit, Cong X. Kang, and Eunjeong Yi
}

\begin{abstract}
The metric dimension $\operatorname{dim}(G)$ of a graph $G$ is the minimum cardinality of a set of vertices such that every vertex of $G$ is uniquely determined by its vector of distances to the chosen vertices. Let $v$ and $e$ respectively denote a vertex and an edge of a graph $G$. We show that, for any integer $k$, there exists a graph $G$ such that $\operatorname{dim}(G-v)-\operatorname{dim}(G)=k$. For an arbitrary edge $e$ of any graph $G$, we prove that $\operatorname{dim}(G-e) \leq \operatorname{dim}(G)+2$. We also prove that $\operatorname{dim}(G-e) \geq \operatorname{dim}(G)-1$ for $G$ belonging to a rather general class of graphs. Moreover, we give an example showing that $\operatorname{dim}(G)-\operatorname{dim}(G-e)$ can be arbitrarily large.
\end{abstract}

KEYWORDS AND PHRASES: Distance, resolving set, metric dimension, vertex deletion, edge deletion.

AMS 2000 SUBJECT CLASSIFICATIONS: 05C12.

\section{Introduction}

Let $G=(V(G), E(G))$ be a simple, undirected, connected, and nontrivial graph with order $|V(G)|$. The degree of a vertex $v$ in $G$, denoted by $\operatorname{deg}_{G}(v)$, is the number of edges that are incident to $v$ in $G$; an end-vertex is a vertex of degree one. We denote by $K_{n}, C_{n}$, and $P_{n}$ the complete graph, the cycle, and the path on $n$ vertices, respectively. The distance between two vertices $v, w \in V(G)$ is denoted by $d_{G}(v, w)$; we drop $G$ if it is clear in the context. For other terminologies in graph theory, we refer to [4].

A vertex $x \in V(G)$ resolves a pair of vertices $u, v \in V(G)$ if $d(u, x) \neq$ $d(v, x)$. A set of vertices $S \subseteq V(G)$ resolves $G$ if every pair of distinct vertices of $G$ is resolved by a vertex in $S$; then $S$ is called a resolving set of $G$. For an ordered set $S=\left\{u_{1}, u_{2}, \ldots, u_{k}\right\} \subseteq V(G)$ of distinct vertices, the metric code (or code, for short) of $v \in V(G)$ with respect to $S$ is the $k$-vector $\operatorname{code}_{S}(v)=\left(d\left(v, u_{1}\right), d\left(v, u_{2}\right), \ldots, d\left(v, u_{k}\right)\right)$. The metric dimension of $G$, denoted by $\operatorname{dim}(G)$, is the minimum of $|S|$ as $S$ varies over all resolving sets of $G$.

Slater $[14,15]$ introduced the concept of a resolving set for a connected graph under the term locating set; he referred to a minimum resolving set as 
a reference set, and the cardinality of a minimum resolving set as the location number of a graph. Independently, Harary and Melter [8] studied these concepts under the term metric dimension. Metric dimension as a graph parameter has numerous applications, among them are robot navigation [10], sonar [14], combinatorial optimization [12], and pharmaceutical chemistry [3]. It was noted in [7] that determining the metric dimension of a graph is an NP-hard problem. Metric dimension has been heavily studied. For a survey on metric dimension and some variations, see [5] by Chartrand and Zhang. For a comparative study of metric dimension and graph parameters of more algebraic flavor, see [1] by Bailey and Cameron.

The question as to the effect of the deletion of a vertex or of an edge on the metric dimension of a graph was raised as a fundamental question in graph theory by Chartrand and Zhang in [5]. We address the question as follows: We show graphs $G$ such that $\operatorname{dim}(G-v)$ is arbitrarily large (or small) relative to $\operatorname{dim}(G)$. For $e \in E(G)$, we prove that $\operatorname{dim}(G-e) \leq \operatorname{dim}(G)+2$ for any graph $G$, and we prove that $\operatorname{dim}(G-e) \geq \operatorname{dim}(G)-1$ for $G$ belonging to a rather general class of graphs. In general, we show that $\operatorname{dim}(G)-\operatorname{dim}(G-e)$ can be arbitrarily large.

\section{The effect of vertex deletion on metric dimension of graphs}

We first recall some basic facts on metric dimension for background.

Theorem 2.1. [3] For a connected graph $G$ of order $n \geq 2$ and diameter $d$,

$$
f(n, d) \leq \operatorname{dim}(G) \leq n-d,
$$

where $f(n, d)$ is the least positive integer $k$ for which $k+d^{k} \geq n$.

A generalization of Theorem 2.1 has been given in [9] by Hernando et al.

Theorem 2.2. [9] Let $G$ be a graph of order $n$, diameter $d \geq 2$, and metric dimension $k$. Then

$$
n \leq\left(\left\lfloor\frac{2 d}{3}\right\rfloor+1\right)^{k}+k \sum_{i=1}^{\left\lceil\frac{d}{3}\right\rceil}(2 i-1)^{k-1}
$$

Theorem 2.3. [3] Let $G$ be a connected graph of order $n \geq 2$. Then

(a) $\operatorname{dim}(G)=1$ if and only if $G=P_{n}$,

(b) $\operatorname{dim}(G)=n-1$ if and only if $G=K_{n}$,

(c) for $n \geq 4, \operatorname{dim}(G)=n-2$ if and only if $G=K_{s, t}(s, t \geq 1), G=$ $K_{s}+\bar{K}_{t}(s \geq 1, t \geq 2)$, or $G=K_{s}+\left(K_{1} \cup K_{t}\right)(s, t \geq 1)$; here, $A+B$ 

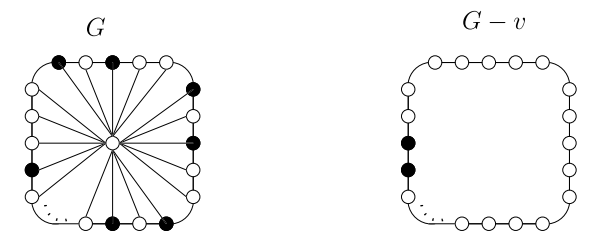

Figure 1: A graph $G$ such that $\operatorname{dim}(G)-\operatorname{dim}(G-v)$ can be arbitrarily large.

denotes the graph obtained from the disjoint union of graphs $A$ and $B$ by joining every vertex of $A$ with every vertex of $B$, and $\bar{C}$ denotes the complement of a graph $C$.

The following definitions are stated in [3]. Fix a graph $G$. A vertex of degree at least three is called a major vertex. An end-vertex $u$ is called a terminal vertex of a major vertex $v$ if $d(u, v)<d(u, w)$ for every other major vertex $w$. The terminal degree of a major vertex $v$ is the number of terminal vertices of $v$. A major vertex $v$ is an exterior major vertex if it has positive terminal degree. Let $\sigma(G)$ denote the sum of terminal degrees of all major vertices of $G$, and let $\operatorname{ex}(G)$ denote the number of exterior major vertices of $G$. Two vertices $u, v \in V(G)$ are called twins if $N(u)-\{v\}=$ $N(v)-\{u\}$, where $N(u)$ is the set of all vertices adjacent to $u$ in $G$. Notice that $S \cap\{u, v\} \neq \emptyset$ if $S$ is a resolving set and $u, v$ are twins for any graph. We now recall two theorems useful in the two examples which follow.

Theorem 2.4. [3, 10, 11] If $T$ is a tree that is not a path, then $\operatorname{dim}(T)=$ $\sigma(T)-\operatorname{ex}(T)$.

Theorem 2.5. [2, 13] For $n \geq 3$, let $W_{1, n}=C_{n}+K_{1}$ be the wheel graph on $n+1$ vertices. Then

$$
\operatorname{dim}\left(W_{1, n}\right)=\left\{\begin{array}{cl}
3 & \text { if } n=3 \text { or } n=6 \\
\left\lfloor\frac{2 n+2}{5}\right\rfloor & \text { otherwise. }
\end{array}\right.
$$

The following example appeared in [2].

Example 2.6. There exists a graph $G$ such that $\operatorname{dim}(G)-\operatorname{dim}(G-v)$ can be arbitrarily large; take $G=W_{1, n}$ for $n \geq 7$ and let $v$ be the central vertex of degree $n$ in $G$ (see Figure 1). Notice that $\operatorname{dim}(G-v)=2$ since $G-v \cong C_{n}$, whereas $\operatorname{dim}(G)=\left\lfloor\frac{2 n+2}{5}\right\rfloor$ by Theorem 2.5 .

Example 2.7. There exists a graph $G$ such that $\operatorname{dim}(G-v)-\operatorname{dim}(G)$ can be arbitrarily large. For $k \geq 6$, let $G-v$ be a tree with $k$ exterior major vertices, $u_{1}, u_{2}, \ldots, u_{k}$, and three terminal vertices $\ell_{i, 1}, \ell_{i, 2}, \ell_{i, 3}$ for each $u_{i}$, 

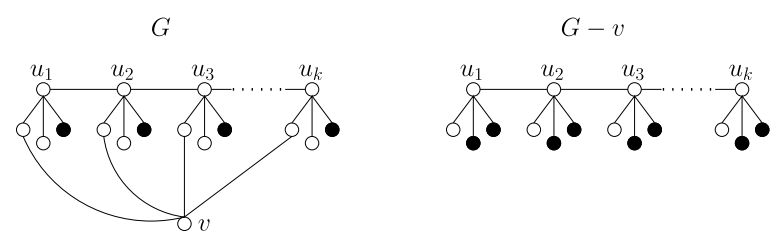

Figure 2: A graph $G$ such that $\operatorname{dim}(G-v)-\operatorname{dim}(G)$ can be arbitrarily large.

where $1 \leq i \leq k$; let $G$ be the graph obtained by joining $\ell_{1,1}, \ell_{2,1}, \ldots, \ell_{k, 1}$ to a new vertex $v$ (see Figure 2). By Theorem 2.4, $\operatorname{dim}(G-v)=2 k$. We will show that $\operatorname{dim}(G)=k$. Since $\ell_{i, 2}$ and $\ell_{i, 3}$ are twins for each $i(1 \leq i \leq k)$ in $G$, $\operatorname{dim}(G) \geq k$. On the other hand, $d_{G}\left(\ell_{i, 3}, v\right)=3$ implies that $d_{G}\left(\ell_{i, 3}, \ell_{i+3,1}\right)=$ 4 and $d_{G}\left(\ell_{i, 3}, \ell_{i+3,2}\right)=5$. So, if $k \geq 6$, then $\left\{\ell_{i, 3} \mid 1 \leq i \leq k\right\}$ forms a resolving set for $G$; thus $\operatorname{dim}(G) \leq k$.

\section{The effect of edge deletion on metric dimension of graphs}

Next, we consider how the metric dimension of a graph changes upon deletion of an edge. The following theorem is stated in [3], with a correct proof given in [6].

Theorem 3.1. [3, 6] Let $T$ be a tree of order at least three. If $e \in E(\bar{T})$, then

$$
\operatorname{dim}(T)-2 \leq \operatorname{dim}(T+e) \leq \operatorname{dim}(T)+1 .
$$

It turns out that the lower bound in the preceding theorem holds for all graphs.

Theorem 3.2. For any graph $G$ and any edge $e \in E(G)$, we have

$$
\operatorname{dim}(G-e) \leq \operatorname{dim}(G)+2 .
$$

Proof. Let $S$ be a minimum resolving set for $G$, and let $u$ and $v$ be the endpoints of the edge $e$. We will show that $S^{\prime}=S \cup\{u, v\}$ is a resolving set for $G-e$. Let $x$ and $y$ be distinct vertices in $V(G-e)=V(G)$ which, in the graph $G$, are resolved by $z \in S$. Suppose $x$ and $y$, in the graph $G-e$, are not resolved by $z$; then $d_{G-e}(x, z)=d_{G-e}(y, z)$. We consider two cases.

Case I. For one of $x$ and $y$, say $y$, the distance to $z$ is not changed by removing edge $e$; so $d_{G-e}(y, z)=d_{G}(y, z)$. In this case, $d_{G}(y, z)=d_{G-e}(y, z)=$ $d_{G-e}(x, z)>d_{G}(x, z)$ and the edge $e$ must lie on every $x-z$ geodesic in $G$. Thus, up to transposing the labels $u$ and $v$, we have $d_{G}(x, u)+d_{G}(u, v)+$ 


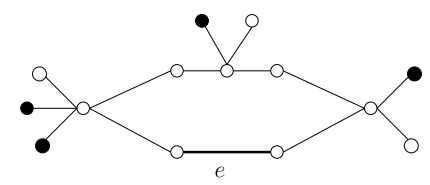

Figure 3: A graph $G$ with $\operatorname{dim}(G-e)=\operatorname{dim}(G)+2$.

$d_{G}(v, z)=d_{G}(x, z)$. Notice that $d_{G}(x, u)=d_{G-e}(x, u)$, since there is an $x-u$ geodesic in $G$ that does not use edge $e$. Since $d_{G}(x, u)+d_{G}(u, z)=$ $d_{G}(x, z)<d_{G}(y, z) \leq d_{G}(y, u)+d_{G}(u, z)$, we must have $d_{G}(x, u)<d_{G}(y, u)$. But then $d_{G-e}(x, u)=d_{G}(x, u)<d_{G}(y, u) \leq d_{G-e}(y, u)$, so $u \in S^{\prime}$ resolves $x$ and $y$.

Case II. For both $x$ and $y$, the distance to $z$ is increased by removing the edge $e$. In this case, the edge $e$ must lie on every $x-z$ geodesic and on every $y-z$ geodesic in $G$. Notice that if a geodesic from some vertex $a$ to another vertex $c$ traverses the edge $e$ in the order $u, v$ (as opposed to $v, u$ ), then a geodesic containing $e$ from any vertex $b$ to $c$ must also traverse $e$ in the order $u, v$ : For the sake of contradiction, let an $a-c$ geodesic have the form $a, \ldots, u, v, \ldots, c$ and let some $b-c$ geodesic have the form $b, \ldots, v, u, \ldots, c$. The presence of the $a-c$ geodesic implies that $d(u, v)+d(v, c)=d(u, c)$, and the presence of the $b-c$ geodesic implies that $d(v, u)+d(u, c)=d(v, c)$. The sum of the two equations simplifies to $d(u, v)=0$, a contradiction. Suppose that $u$ is traversed before $v$ by a $x-z$ geodesic and a $y-z$ geodesic (directed towards $z$ ) in $G$, then a $x-u$ geodesic and a $y-u$ geodesic, neither containing the edge $e$, are obtained by truncating a common $u-z$ geodesic in $G$; thus, $u$ resolves $x$ and $y$ in $G-e$. To complete the proof, simply swap the letters $u$ and $v$ in the preceding sentence.

Example 3.3. For the sharpness of the upper bound of Theorem 3.2, see Figure 3. Notice that $\operatorname{dim}(G)=4$ (the solid vertices in Figure 3 form a minimum resolving set of $G)$. By Theorem $2.4, \operatorname{dim}(G-e)=6$, and hence $\operatorname{dim}(G-e)=\operatorname{dim}(G)+2$.

Next, we consider how small the metric dimension of $G$ could become upon deleting an edge of $G$. The following theorem is really an example; we are calling it a theorem in deference to its importance and the effort expended in its discovery!

Theorem 3.4. There exists a graph $G$ such that $\operatorname{dim}(G)-\operatorname{dim}(G-e)$ can be arbitrarily large. Let $G$ be the graph in Figure 4 for $k \geq 2$, and let $e=A B \in E(G)$. Then $\operatorname{dim}(G)=2 k$ and $\operatorname{dim}(G-e)=k+1$. 


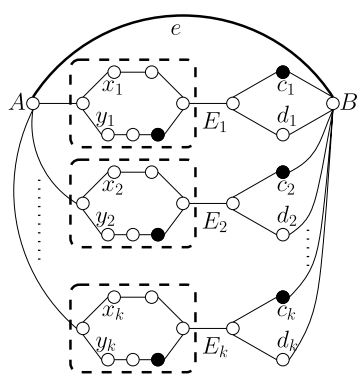

G

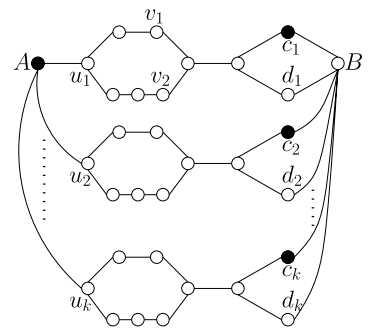

$G^{\prime}=G-e$

Figure 4: A graph $G$ such that $\operatorname{dim}(G)-\operatorname{dim}(G-e)$ can be arbitrarily large.

Proof. Let $S$ be a minimum resolving set for $G$, and let $S^{\prime}$ be a minimum resolving set for $G^{\prime}=G-e$. Notice that, for each $i(1 \leq i \leq k),\left|S \cap\left\{c_{i}, d_{i}\right\}\right| \geq$ 1 since $c_{i}$ and $d_{i}$ are twin vertices in $G$; similarly, $\left|S^{\prime} \cap\left\{c_{i}, d_{i}\right\}\right| \geq 1$. Without loss of generality, we may assume $S_{0}=\left\{c_{i} \mid 1 \leq i \leq k\right\} \subseteq S \cap S^{\prime}$. For the sake of complete clarity, let $\operatorname{code}_{S}(x, G)$ denote the code vector of $x$ with respect to the set of vertices $S$ in the graph $G$.

First, we show that $\operatorname{dim}(G)=2 k$. Notice that, for each $i(1 \leq i \leq k)$, code $_{S_{0}}\left(x_{i}, G\right)=\operatorname{code}_{S_{0}}\left(y_{i}, G\right)$. Further, if $S \cap E_{i}=\emptyset$ for some $i$, then $\operatorname{code}_{S}\left(x_{i}, G\right)=\operatorname{code}_{S}\left(y_{i}, G\right)$, contradicting the assumption that $S$ is a resolving set for $G$, and thus $\left|S \cap E_{i}\right| \geq 1$ for each $i(1 \leq i \leq k)$. So, $\operatorname{dim}(G) \geq 2 k$. Since the solid vertices of $G$ in Figure 4 form a resolving set for $G, \operatorname{dim}(G)=2 k$.

Next, we show that $\operatorname{dim}\left(G^{\prime}\right)=k+1$. Since, for instance, $\operatorname{code}_{S_{0}}\left(v_{1}, G^{\prime}\right)=$ code $_{S_{0}}\left(v_{2}, G^{\prime}\right)$, we have $\left|S^{\prime}-S_{0}\right| \geq 1$, implying that $\operatorname{dim}\left(G^{\prime}\right) \geq k+1$. Since $\{A\} \cup S_{0}$ forms a resolving set for $G^{\prime}, \operatorname{dim}\left(G^{\prime}\right)=k+1$.

In [6], it's proved that $\operatorname{dim}(G+e) \leq \operatorname{dim}(G)+1$ when $G$ is a tree; a key idea used there is the notion of "strong resolution", identified but not named in the paper [11] by Poisson and Zhang: we say vertices $u$ and $v$ are strongly resolved by a set of vertices $W$ if $\operatorname{code}_{W}(u)-\operatorname{code}_{W}(v) \neq(a, \ldots, a)$ for any $a \in \mathbb{Z}$. In fact, the proof in $[6]$ shows that $\operatorname{dim}(G+e) \leq \operatorname{dim}(G)+1$ holds for a more general class of graphs than just trees.

Theorem 3.5. Suppose there exists an induced cycle $C$ in $G+e$ which contains the edge $e$, with the vertices of $C$ cyclically labeled as $c_{0}, \ldots, c_{n-1}$. Let $G_{i}$ be the subgraph of $G+$ e rooted at $c_{i}$; i.e., $G_{i}$ is the maximal subgraph of $G+e$ such that $c_{i} \in V\left(G_{i}\right)$ and $E\left(G_{i}\right) \cap\left\{c_{i-1} c_{i}, c_{i} c_{i+1}\right\}=\emptyset$ (the indices 


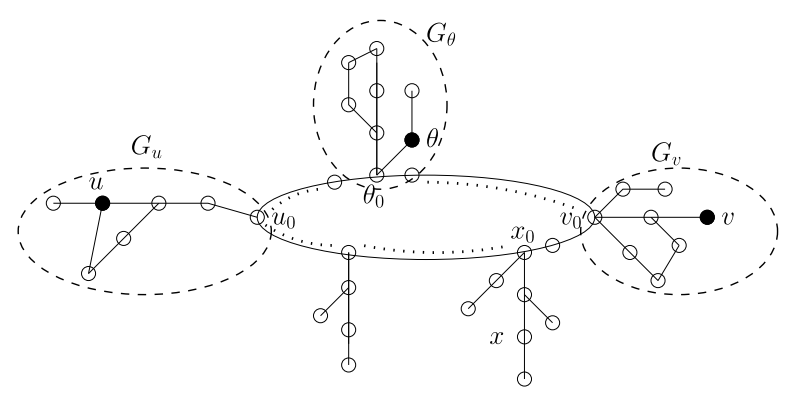

Figure 5: The set $\{u, v, \theta\}$ resolves the subgraphs $G_{i}$ 's, $i \in\{u, v, \theta\}$, from each other, where two of $\left\{u_{0}, v_{0}, \theta_{0}\right\} \subseteq\left\{c_{0}, c_{1}, \ldots, c_{n-1}\right\}$ attain the diameter of the cycle $C$.

of vertices being taken modulo $n)$. Suppose further that $V\left(G_{i}\right) \cap V\left(G_{j}\right)=\emptyset$ for $i \neq j$. Then $\operatorname{dim}(G+e) \leq \operatorname{dim}(G)+1$ (see Figure 5$)$.

Proof. Exactly as in [6]; see Appendix A.

Definition 3.6. We say a "graph $G$ has no even cycles" if, whenever there exists a (not necessarily induced) subgraph of $G$ isomorphic to a cycle $C_{n}$, $n$ must be an odd integer.

Lemma 3.7. Suppose $G$ has no even cycles; then any two (odd) cycles of $G$ intersect in at most one vertex.

Proof. Suppose two cycles $A^{\prime}$ and $B^{\prime}$ share two distinct vertices $u$ and $v$. Then there exist two cycles $A$ and $B$ and a fixed $u-v$ path $P^{2}$ such that $A$ is the concatenation of a path $P^{1}$ with $P^{2}$ and $B$ is the concatenation of a path $P^{3}$ with $P^{2}$. Since the length of $A$ is odd, the length of $P^{1}$ and the length of $P^{2}$ must have opposite parity. Thus, either the concatenation of $P^{3}$ with $P^{1}$ or the concatenation of $P^{3}$ with $P^{2}$ forms an even cycle, and we have a contradiction.

Thus, we have the following

Corollary 3.8. Suppose that a connected graph $G$ has no even cycles; then the following results hold: (1) Every cycle occurring as a subgraph of $G$ occurs as an induced subgraph of $G$; (2) There is a unique geodesic between any pair of vertices of $G$; (3) $\operatorname{dim}(G-e) \geq \operatorname{dim}(G)-1$.

Proof. Parts (1) and (2) readily follow from Lemma 3.7. To obtain part (3), apply part (1) of the present corollary, Lemma 3.7, and Theorem 3.5 to $G$. 


\section{Appendix A. Proof of Theorem 3.5}

The following is an excerpt from reference [6] (by Eroh, Kang, and $\mathrm{Yi}$; arXiv:1408.5943); we post it herewith so that the present paper is self-contained.

The cycle rank of a graph $G$, denoted by $r(G)$, is defined as $|E(G)|-$ $|V(G)|+1$. For a tree $T, r(T)=0$. If a graph $G$ has $r(G)=1$, we call it a unicyclic graph. By $T+e$, we shall mean a unicyclic graph obtained from a tree $T$ by attaching a new edge $e \in E(\bar{T})$. In [11], the notion of a resolving set $W$ with the property $\operatorname{code}_{W}(u)-\operatorname{code}_{W}(v) \neq(a, \ldots, a)$ for any $a \in \mathbb{Z}$ was identified and shown to be very useful. We will say that " $G$ is strongly resolved by $W$ " if $\operatorname{code}_{W}(u)-\operatorname{code}_{W}(v) \neq(a, \ldots, a)$ for any $a \in \mathbb{Z}$ and any $u, v \in V(G)$. Still following [11], observe that $u \sim_{W} v$ if and only if $\operatorname{code}_{W}(u)-\operatorname{code}_{W}(v)=(a, \ldots, a)$ for some $a \in \mathbb{Z}$ defines an equivalence relation $\sim_{W}$ on $V(G)$; let $[u]_{W}$ denote the equivalence class of $u$ under this relation.

Theorem A.1. [3] If $T$ is a tree of order at least three and $e$ is an edge of $\bar{T}$, then

$$
\operatorname{dim}(T+e) \leq \operatorname{dim}(T)+1 .
$$

Proof (as in [6]). The claim holds when $T$ is a path $P_{n}$, as the two endvertices of $P_{n}$ form a basis (minimum resolving set) for $P_{n}+e$ : If $e=v_{i} v_{j}$ where $i<j$, then $v_{i}$ and $v_{j}$, being adjacent vertices, resolve vertices on the unique cycle $C$ of $P_{n}+e$ among themselves (whence we say " $v_{i}$ and $v_{j}$ resolve $C$ "). But then $W=\left\{v_{1}, v_{n}\right\}$ resolves $C$ since for any $v \in V(C)$, $\operatorname{code}_{W^{\prime}}(v)=\operatorname{code}_{W}(v)+\left(a_{1}, a_{2}\right)$, where $W^{\prime}=\left\{v_{i}, v_{j}\right\}$ and $\left(a_{1}, a_{2}\right)$ is a fixed vector. Further, $v_{1}$ and $v_{n}$ obviously resolve vertices in $V\left(P_{n}+e\right)-V(C)$ among themselves and from $V(C)$.

So, let $T$ be a tree which is not a path, and thus $\operatorname{dim}(T) \geq 2$. Cyclically label the vertices lying on the unique cycle $C$ of $T+e(e \in E(\bar{T}))$ by $u_{1}, \ldots u_{k}(k \geq 3)$. Denote by $T_{i}$ the subtree rooted at $u_{i}$ (in other words, the component of $(T+e)-E(C)$ which contains $\left.u_{i}\right)$. Given any basis $B$ of $T$, partition $B$ into the disjoint union of sub-bases $B_{i}$, where $B_{i} \subseteq V\left(T_{i}\right)$, $1 \leq i \leq k$; assume, without loss of generality, that $B_{1} \neq \emptyset$. If $B_{i}=\emptyset$ for each $i \neq 1$, then $T-T_{1}$ must be a path (for $B$ to be a basis of $T$ ); in this case, either $B \cup\left\{u_{2}\right\}$ or $B \cup\left\{u_{k}\right\}$ is a resolving set for $T+e$.

So, assume there exists $1<i \leq k$ such that $B_{i} \neq \emptyset$. If there exist two non-empty sub-bases $B_{i}$ and $B_{j}$ such that $d_{T+e}\left(u_{i}, u_{j}\right)=m=\left\lfloor\frac{k}{2}\right\rfloor$, then let $b_{0} \in V(C)-\left\{u_{i}, u_{j}\right\}$ and put $B_{0}=\left\{b_{i}, b_{j}, b_{0}\right\}$ (also put $B_{0}^{\prime}=\left\{u_{i}, u_{j}, b_{0}\right\}$ ) 


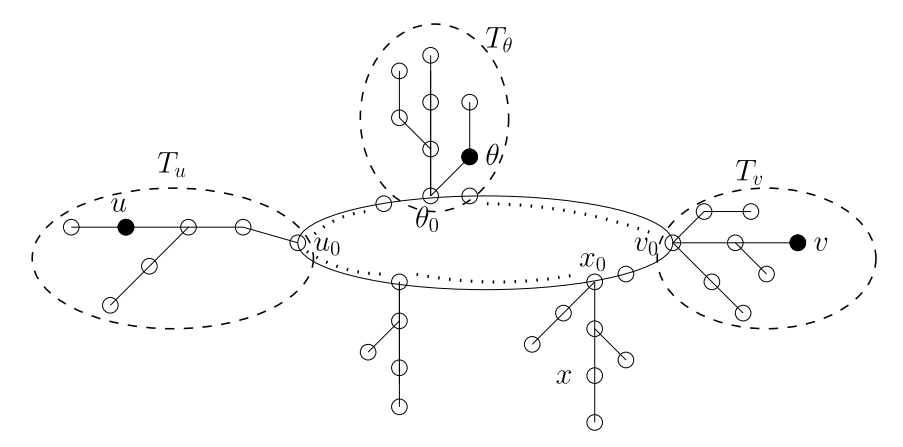

Figure 6: The set $\{u, v, \theta\}$ resolves the subtrees $T_{i}$ 's from each other.

where $b_{i} \in B_{i}$ and $b_{j} \in B_{j}$; otherwise, let $b_{0}=u_{m+1}$ and put $B_{0}=\left\{b_{1}, b_{0}, b_{s}\right\}$ (also put $B_{0}^{\prime}=\left\{u_{1}, b_{0}, u_{s}\right\}$ ), where $b_{1} \in B_{1}$ and $b_{s} \in B_{s} \neq \emptyset$ for some $s \neq 1, m+1$. (The point here is to arrange a resolving set for $T+e$ that contains elements in three subtrees (the $T_{i}$ 's), two of which having roots (the $u_{i}$ 's) attaining the diameter of the cycle $C$.) We will show that the set $\widetilde{B}=B \cup\left\{b_{0}\right\}$ is a resolving set for $T+e$. Notice that $B_{0} \subseteq \widetilde{B}$.

By Lemma A.2, we have $\operatorname{code}_{B_{0}}\left(x_{i}\right) \neq \operatorname{code}_{B_{0}}\left(x_{j}\right)$ and, a fortiori, code $_{\widetilde{B}}\left(x_{i}\right) \neq \operatorname{code}_{\widetilde{B}}\left(x_{j}\right)$ for $x_{i} \in V\left(T_{i}\right)$ and $x_{j} \in V\left(T_{j}\right)$, when $i \neq j$. It thus suffices to show that $\forall x, y \in V\left(T_{i}\right)$ where $1 \leq i \leq k, \operatorname{code}_{\widetilde{B}}(x) \neq$ code $_{\widetilde{B}}(y)$. Accordingly, let $x, y \in V\left(T_{i}\right)$ be given for a fixed $i$. It's clear that if $d_{T}(x, b) \neq d_{T}(y, b)$ for some $b \in B_{i}$, then $d_{T+e}(x, b) \neq d_{T+e}(y, b)$; so, let $b \in B_{j}$ for some $j \neq i$. Notice that there exists a fixed $a \in \mathbb{N}$ such that $\forall x \in V\left(T_{i}\right), d_{T+e}(x, b)=d_{T}(x, b)-a$. Thus, $d_{T}(x, b) \neq d_{T}(y, b)$ implies $d_{T+e}(x, b) \neq d_{T+e}(y, b)$ for $b \notin B_{i}$ as well.

We have thus proved the theorem.

The following lemma shows that subtrees are distinguished by the $B_{0}$ chosen above; see Figure 6 for an illustration of the situation under consideration.

Lemma A.2. Let $B_{0}$ and $B_{0}^{\prime}$ be chosen as in the Proof of Theorem A.1; explicitly, let $B_{0}=\{u, v, \theta\}$ and $B_{0}^{\prime}=\left\{u_{0}, v_{0}, \theta_{0}\right\} \subseteq V(C)$, where $d\left(u_{0}, v_{0}\right)=$ $\operatorname{diam}(C)$ and $u\left(v, \theta\right.$, respectively) is a vertex on the subtree rooted at $u_{0}$ $\left(v_{0}, \theta_{0}\right.$, respectively). Then, we have $\operatorname{code}_{B_{0}}(x) \neq \operatorname{code}_{B_{0}}(y)$ for vertices $x$ and $y$ belonging to distinct subtrees rooted at vertices of the unique cycle $C$ of $T+e$.

Proof. Observe that $B_{0}^{\prime}$ strongly resolves the unique cycle $C$ of $T+e$, because no vertex of $C$ can have shorter distance, by the same value, to 
all vertices of $B_{0}^{\prime}$ than another vertex of $C$. Thus, $B_{0}$ strongly resolves $C$, because there exists a fixed vector $\left(a_{1}, a_{2}, a_{3}\right)$ such that $\forall x \in V(C)$, code $_{B_{0}}(x)=\operatorname{code}_{B_{0}^{\prime}}(x)+\left(a_{1}, a_{2}, a_{3}\right)$. If $x \in V\left(T_{i}\right)$ where $V\left(T_{i}\right) \cap B_{0}=\emptyset$, then $[x]_{B_{0}}=\left[x_{0}\right]_{B_{0}}$, where $x_{0}$ is the root of $T_{i}$ : this is because any path from $x$ of such a subtree $T_{i}$ to a vertex in $B_{0}$ must go through $x_{0}$. Thus $[x]_{B_{0}} \neq[y]_{B_{0}}$ and, a fortiori, $\operatorname{code}_{B_{0}}(x) \neq \operatorname{code}_{B_{0}}(y)$ for $x$ and $y$ belonging to distinct subtrees which have empty intersection with $B_{0}$. If $B_{0}=B_{0}^{\prime}$, then the same reasoning applies to the subtrees containing elements of $B_{0}$. Otherwise, if suffices to check $\operatorname{code}_{B_{0}}(x) \neq \operatorname{code}_{B_{0}}(y)(1)$ for $x \in V\left(T_{i}\right)$ and $y \in V\left(T_{u}\right)$, (2) for $x \in V\left(T_{i}\right)$ and $y \in V\left(T_{\theta}\right)$, (3) for $x \in V\left(T_{u}\right)$ and $y \in V\left(T_{v}\right)$, and (4) for $x \in V\left(T_{u}\right)$ and $y \in V\left(T_{\theta}\right)$; here $T_{u}, T_{v}, T_{\theta}$, and $T_{i}$ are the subtrees containing $u, v, \theta$, and none of $B_{0}$, respectively. Since the same argument works for all four inequalities, we will only explicitly verify (1).

Suppose, for the sake of contradiction, $\operatorname{code}_{B_{0}}(y)=\operatorname{code}_{B_{0}}(x)$; i.e., $(d(y, u), d(y, v), d(y, \theta))=(d(x, u), d(x, v), d(x, \theta))$ for vertices $y \in V\left(T_{u}\right)$ and $x \in V\left(T_{i}\right)$. Equating the first two coordinates and expanding, we get $d(y, u)=d\left(x, x_{0}\right)+d\left(x_{0}, u_{0}\right)+d\left(u_{0}, u\right)$ and $d\left(y, u_{0}\right)+d\left(u_{0}, v_{0}\right)+d\left(v_{0}, v\right)=$ $d\left(x, x_{0}\right)+d\left(x_{0}, v_{0}\right)+d\left(v_{0}, v\right)$, where $x_{0}$ is the root of the subtree containing $x$. Subtracting the two equations and rearranging terms, we get $d(y, u)=d\left(y, u_{0}\right)+d\left(x_{0}, u_{0}\right)+d\left(u_{0}, u\right)+d\left(u_{0}, v_{0}\right)-d\left(x_{0}, v_{0}\right)$. Now, since $d\left(u_{0}, v_{0}\right)=\operatorname{diam}(C)$, we have $d\left(u_{0}, v_{0}\right)-d\left(x_{0}, v_{0}\right)=d\left(u_{0}, x_{0}\right)$. And we have $d(y, u)=d\left(y, u_{0}\right)+d\left(u_{0}, u\right)+2 d\left(u_{0}, x_{0}\right)$. Since $x \in V\left(T_{i}\right)$ and $T_{i} \neq T_{u}$, $d\left(u_{0}, x_{0}\right)>0$, and we have $d(y, u)>d\left(y, u_{0}\right)+d\left(u_{0}, u\right)$, violating the triangle inequality which $d(\cdot, \cdot)$ must satisfy as a metric.

Remark A.3. Notice that Lemma A.2 still holds if each "subtree $T_{i}$ rooted at $u_{i}$ " is replaced by "subgraph $G_{i}$ rooted at $u_{i}$ " with $G_{i}$ and $G_{j}$ disjoint for $i \neq j$.

\section{References}

[1] R. F. Bailey and P. J. Cameron (2011). Base size, metric dimension and other invariants of groups and graphs. Bull. London Math. Soc. 43(2) 209-242. MR2781204

[2] P. S. Buczkowski, G. Chartrand, C. Poisson and P. Zhang (2003). On $k$-dimensional graphs and their bases. Period. Math. Hungar. 46(1) 9-15. MR1975342

[3] G. Chartrand, L. Eroh, M. A. Johnson and O. R. Oellermann (2000). Resolvability in graphs and the metric dimension of a graph. Discrete Appl. Math. 105 99-113. MR1780464 
[4] G. Chartrand and P. Zhang (2004). Introduction to graph theory. McGraw-Hill, Kalamazoo, MI.

[5] G. Chartrand and P. Zhang (2003). The theory and applications of resolvability in graphs. A Survey. Congr. Numer. 160 47-68. MR2049102

[6] L. Eroh, C. X. Kang and E. Yi. A Comparison between the Metric Dimension and Zero Forcing Number of Trees and Unicyclic Graphs. arXiv:1408.5943.

[7] M. R. Garey and D. S. Johnson (1979). Computers and intractability: A guide to the theory of NP-completeness. Freeman, New York. MR0519066

[8] F. Harary and R. A. Melter (1976). On the metric dimension of a graph. Ars Combin. 2 191-195. MR0457289

[9] C. Hernando, M. Mora, I. M. Pelayo, C. Seara and D. R. Wood (2010). Extremal graph theory for metric dimension and diameter. Electron. J. Combin. 17(1) \#R30. MR2595490

[10] S. Khuller, B. Raghavachari and A. Rosenfeld (1996). Landmarks in graphs. Discrete Appl. Math. 70 217-229. MR1410574

[11] C. Poisson and P. Zhang (2002). The metric dimension of unicyclic graphs. J. Combin. Math. Combin. Comput. 40 17-32. MR1887964

[12] A. Sebö and E. Tannier (2004). On metric generators of graphs. Math. Oper. Res. 29 383-393. MR2065985

[13] B. Shanmukha, B. Sooryanarayana and K. S. Harinath (2002). Metric dimension of wheels. Far East J. Appl. Math. 8(3) 217-229. MR1944130

[14] P. J. Slater (1975). Leaves of trees. Congress. Numer. 14 549-559. MR0422062

[15] P. J. Slater (1998). Dominating and reference sets in a graph. J. Math. Phys. Sci. 22 445-455. MR0966610

LINDA EROH

UniVERSITY OF Wisconsin OSHKOSH

Oshkosh, WI 54901

USA

E-mail address: eroh@uwosh.edu 
Paul Feit

The University of Texas of the Permian Basin

OdEssa, TX 79762

USA

E-mail address: feit_p@utpb.edu

Cong X. KANG

Texas A\&M University at Galveston

Galveston, TX 77553

USA

E-mail address: kangc@tamug.edu

EUNJEONG Yi

TeXas A\&M University at Galveston

Galveston, TX 77553

USA

E-mail address: yie@tamug.edu

Received 22 August 2012 